\title{
La Enseñanza de Conceptos en Biotecnología a través de un Experimento Sencillo y Económico
}

\author{
María V. Curia, Oriana D'Alessandro y Laura E. Briand \\ Universidad Nacional de La Plata, Facultad de Ciencias Exactas, Centro de Investigación y \\ Desarrollo en Ciencias Aplicadas Dr. J. J. Ronco, Centro Científico Tecnológico La Plata, \\ CONICET. Calle $47 \mathrm{~N}^{0}$ 257, B1900AJK La Plata, Buenos Aires-Argentina. (e-mail: \\ briand@quimica.unlp.edu.ar)
}

Recibido Dic. 02, 2009; Aceptado Feb. 16, 2010; Versión Final recibida 08 Mar. 2010

\section{Resumen}

Se propone una experiencia sencilla que es aplicable a la enseñanza de la biotecnología en los niveles primario, secundario y universitario. El experimento consiste en la fermentación de azúcar con levaduras comerciales. En este proceso se genera dióxido de carbono que se recoge en un globo, como indicador visual de la evolución de la fermentación. Este experimento es una vía para que los docentes transmitan una variedad de conceptos de biología, física, química y de procesos biotecnológicos. Las aplicaciones de este experimento de fermentación a través de microorganismos brinda un nexo directo para que los estudiantes puedan entender intuitivamente procesos comunes y domésticos como el leudado de una masa o la producción de bebidas alcohólicas.

Palabras clave: biotecnología, fermentación, levaduras, ciencias naturales, enseñanza.

\section{A Simple and Low Cost Experiment as a Tool to Teach Concepts in Biotechnology}

\begin{abstract}
A simple experiment that can be applied to the teaching of biotechnology to students of either elementary, high school or university level is proposed. The experiment consists of the fermentation of sugar with commercial yeast. This process produces carbon dioxide that is collected in a balloon as a visual indicator of the progress of the fermentation reaction. This particular experiment is a good method to teach various concepts related with chemistry, physics, biology and biotechnology. The applications of the proposed fermentation experiment using microorganisms represents a direct connection for the students to understand common and domestic processes such as dough rising or production of alcoholic beverages.
\end{abstract}

Keywords: biotechnology, fermentation, yeasts, natural science, teaching 


\section{INTRODUCCIÓN}

La biotecnología, definida como la aplicación de un fenómeno biológico para crear productos y servicios, es una de las ramas de la tecnología que mayor crecimiento y popularidad ha cobrado en nuestra sociedad (Braun y Moses, 2004). El constante desarrollo de tecnología basada en la biocatálisis demandará un número creciente de profesionales capacitados en ese área por lo cual, resulta fundamental que los alumnos de todos los niveles educativos y el público en general estén familiarizados con los principios más básicos de la biotecnología a través de productos y procesos de la vida cotidiana. Por otro lado, la enseñanza de las ciencias en todos los niveles debe estar orientada al entendimiento del mundo en el que vivimos a través de la comprensión, interpretación y explicación de fenómenos naturales (Bravo, 2009). En este sentido, los autores han presentado el experimento de fermentación que se describe en este trabajo, en la $36^{\circ}$ Feria Internacional del Libro (22 de abril al 10 de mayo de 2009 en Buenos Aires, Argentina) y se ha demostrado que un experimento sencillo atrae la atención de público de todas las edades. En el contexto internacional de la popularización del conocimiento se implementaron programas como el European Initiative for Biotechnology Education y Educating the European Public for Biotechnology cuyo objetivo es promover el entendimiento y el debate público de la biotecnología (Braun y Moses 2004). Con el mismo propósito se desarrolla la REDBIO en Latinoamérica y el Caribe que cuenta con el apoyo de la Organización de las Naciones Unidas para la Agricultura y Alimentación (Rota e Izquierdo, 2003). Esta red desarrolló un curso para la enseñanza de la biotecnología orientada a la agricultura que se implementó en escuelas primarias de Brasil. Esta interesante iniciativa utilizó una historieta como herramienta pedagógica.

Desde el punto de vista de la educación universitaria, se destaca el programa de biotecnología que se implementó en el Moorpark College (California, USA) con el apoyo de las industrias locales (Harrigan, 2003). Este programa que tiene como finalidad entrenar a los alumnos en tópicos tales como, fermentación microbiana, cultivo celular, recuperación y purificación de productos entre otros, fue desarrollado por docentes, gerentes y científicos de diversas industrias dedicadas a biomanufactura. Más recientemente, se ha propuesto la utilización de programas de computación especialmente diseñados para enseñar biotecnología en el contexto del método TEL, Technology Enhanced Learning (Winters y Mor, 2008). En el presente trabajo se describe una experimentación sencilla basada en el proceso de fermentación con levaduras como metodología para transmitir conceptos básicos de biocatálisis y biotecnología a los alumnos del nivel educativo primario y secundario, como así también a los de los primeros años de la universidad.

\section{FUNDAMENTOS TEÓRICOS}

Las levaduras son hongos unicelulares, la mayoría perteneciente a los Ascomycetos. Normalmente son ovales, esféricas o casi cilíndricas. Dentro del género Saccharomyces, la especie cerevisiae constituye la levadura y el microorganismo eucariota más estudiado. Se la puede encontrar en varios materiales ricos en azúcar como zumo de fruta y néctar (Madigan, 2009). Las levaduras son los microorganismos más importantes y más ampliamente utilizados en la industria. Se cultivan por sus propias células, por sus componentes celulares y por los productos finales que producen. Pueden llevar a cabo dos tipos de metabolismos quimiorganotróficos: la fermentación y la respiración. Cuando el oxigeno está presente, las levaduras crecen eficazmente sobre el azúcar formando biomasa y $\mathrm{CO}_{2}$ (respiración) (ec. 1). Sin embargo en ausencia de oxígeno las levaduras cambian a un metabolismo anaeróbico (fermentación) que origina menor cantidad de biomasa celular pero cantidades notables de alcohol y $\mathrm{CO}_{2}$ (ec. 2).

$\mathrm{C}_{6} \mathrm{H}_{12} \mathrm{O}_{6(\mathrm{aq})}+6 \mathrm{O}_{2(\mathrm{~g})} \longrightarrow 6 \mathrm{CO}_{2(\mathrm{~g})}+6 \mathrm{H}_{2} \mathrm{O}$

(1)

$\mathrm{C}_{6} \mathrm{H}_{12} \mathrm{O}_{6(\mathrm{aq})} \longrightarrow 2 \mathrm{CH}_{3} \mathrm{CH}_{2} \mathrm{OH}+2 \mathrm{CO}_{2(\mathrm{~g})}$

En la producción del pan y la mayoría de las bebidas alcohólicas, se utiliza la levadura Saccharommyces cerevisiae para producir etanol y $\mathrm{CO}_{2}$. En la producción de vino, cuando se prensan las uvas para obtener el mosto, un pequeño número de células de levaduras presentes 
en las uvas, ya desde las viñas, se transfieren al mosto. Durante los primeros días del proceso de elaboración del vino, las levaduras crecen por respiración consumiendo oxigeno. Tan pronto como se acaba el oxígeno comienza la fermentación y con ello el proceso de formación de alcohol a partir de la glucosa. Este cambio de metabolismo aeróbico al anaeróbico es crítico. Las levaduras también se usan como agentes fermentadores para levantar la masa en la elaboración del pan y conferirle el aroma que lo caracteriza. En este caso lo importante no es el alcohol sino el $\mathrm{CO}_{2}$, el otro producto de la fermentación alcohólica. Durante el proceso de leudado, la levadura se mezcla con la masa húmeda en presencia de una pequeña cantidad de azúcar. Así la levadura convierte el azúcar en alcohol y $\mathrm{CO}_{2}$, a continuación el $\mathrm{CO}_{2}$ gaseoso se expande y hace que la masa se levante y se esponje. Cuando se cuece el pan, el calor expulsa el $\mathrm{CO}_{2}$ y el alcohol, por lo que se forman agujeros dentro de la masa que le dan su textura ligera característica. En el experimento que se detallará en la próxima sección se demostrará el crecimiento aeróbico de la levadura a través de la generación de dióxido de carbono gaseoso y la posterior fermentación del azúcar, que se detectará por el caracte-rístico olor del etanol. La reproducción de las levaduras puede ser asexual (por gemación y fisión) y sexual (por ascósporas). La división es, casi siempre, por gemación, en este proceso, la nueva célula se forma como un pequeño bulto en la célula madre que crece hasta separarse de ella. Algunas levaduras poseen reproducción sexual por conjugación en la que se fusionan dos células. La célula resultante es un zigoto verdadero y de él emergen esporas sexuales por reducción meiótica.

\section{METODOLOGÍA}

Los materiales a utilizar son: levadura prensada que se adquiere en los supermercados, azúcar, un globo, una botella de aproximadamente $200 \mathrm{ml}$, un cristalizador y agua a $37{ }^{\circ} \mathrm{C}$. La figura 1 presenta las etapas del experimento y a continuación se describen la metodología a seguir para la utilización de levaduras como biocatalizadores en la reacción de fermentación de azúcar: (a) disolver dos cucharaditas de levadura en un poco de agua caliente y agregar una cucharada de azúcar; (b) transferir el líquido a la botella y colocarla en un recipiente con agua caliente y (c) colocar el globo en el pico de la botella, manteniendo la temperatura del agua durante todo el ensayo. A medida que transcurre el tiempo, el globo se infla debido a que las levaduras utilizan el azúcar como alimento y en el proceso liberan dióxido de carbono, el cual infla el globo (Fig. 1d). Durante los primeros minutos del ensayo estos microorganismos unicelulares crecen por respiración consumiendo oxigeno, pero cuando éste se acaba comienza la degradación del azúcar mediante fermentación y con ello el proceso de formación de alcohol a partir de la glucosa.

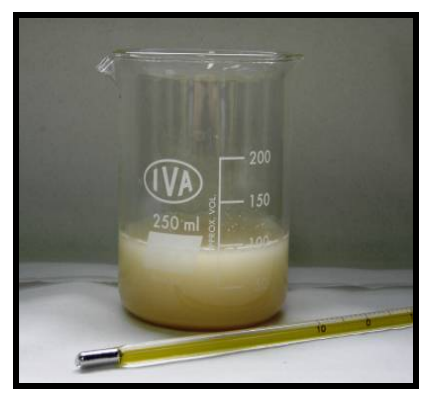

(a)

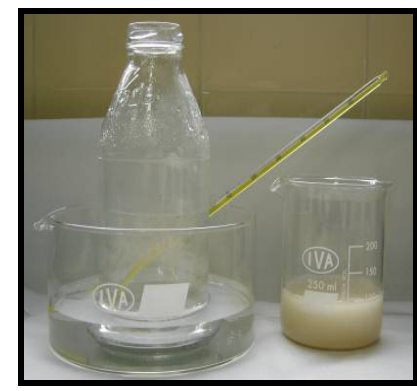

(b)

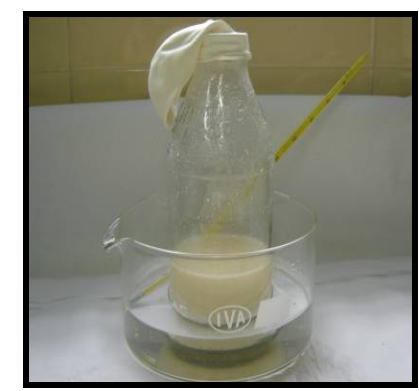

(c)

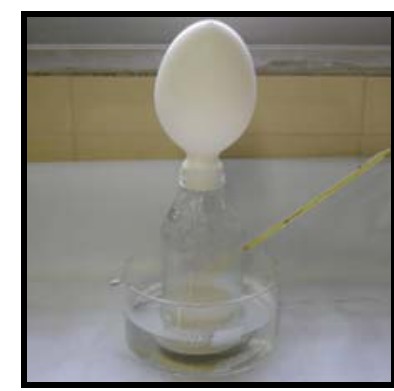

(d)

Fig. 1: Etapas del experimento de fermentación de azúcar con levadura prensada.

\section{RESULTADOS}

El experimento descrito en la sección anterior no solamente es simple y de muy bajo costo, sino que es una herramienta para que el docente transmita los aspectos teóricos básicos de la química, física, termodinámica y biología según puede observarse en la Tabla 1. La figura 2 por otro lado muestra la división de las células de levadura por gemación a través de la observación de levaduras disueltas en agua (en una solución diluida y sin agregado de azúcar) en un microscopio electrónico de barrido. Adicionalmente es posible ampliar el experimento a través de la inmovilización de las levaduras en esferas de gel de alginato de calcio para una posterior fermentación del azúcar (Jenkins, 2000). En este contexto, el docente podrá demostrar que la 
inmovilización redunda en el mejoramiento del bio-proceso porque el biocatalizador en esas condiciones es reutilizable.

Tabla 1: Metodología pedagógica para la integración de las observaciones experimentales y los conceptos teóricos involucrados en el experimento de fermentación con levaduras.

\begin{tabular}{|l|l|}
\hline \multicolumn{1}{|c|}{ Área y temática específica } & \multicolumn{1}{c|}{ Metodología pedagógica } \\
\hline $\begin{array}{l}\text { Química básica, formulación y } \\
\text { balanceo de ecuaciones químicas }\end{array}$ & $\begin{array}{l}\text { Planteo de las ecuaciones químicas relacionadas con los } \\
\text { metabolismos de la levadura }\end{array}$ \\
\hline Termodinámica, termoquímica & $\begin{array}{l}\text { Cálculo de calores de reacción de las reacciones de oxidación } \\
\text { total y parcial de glucosa a partir de las entalpías de formación } \\
\text { estándar }\end{array}$ \\
\hline $\begin{array}{l}\text { Biología, características } \\
\text { morfológicas de las levaduras }\end{array}$ & $\begin{array}{l}\text { Observación de las levaduras en un microscopio óptico. Se realiza } \\
\text { una tinción simple con azul de metileno de una solución acuosa } \\
\text { de levadura }\end{array}$ \\
\hline $\begin{array}{l}\text { Biología, mecanismo de reproduc- } \\
\text { ción de las levaduras por } \\
\text { gemación }\end{array}$ & $\begin{array}{l}\text { A través de la observación de las levaduras en un microscopio } \\
\text { electrónico de barrido (ver foto) }\end{array}$ \\
\hline $\begin{array}{l}\text { Física, la ecuación de estado de } \\
\text { los gases ideales }\end{array}$ & $\begin{array}{l}\text { Es posible determinar el volumen de } \mathrm{CO}_{2(\mathrm{~g})} \text { al recogerlo en una } \\
\text { campana para gases preparada con una probeta. A partir de la } \\
\text { ecuación de estado se calcula el número de moles de dióxido. }\end{array}$ \\
\hline
\end{tabular}

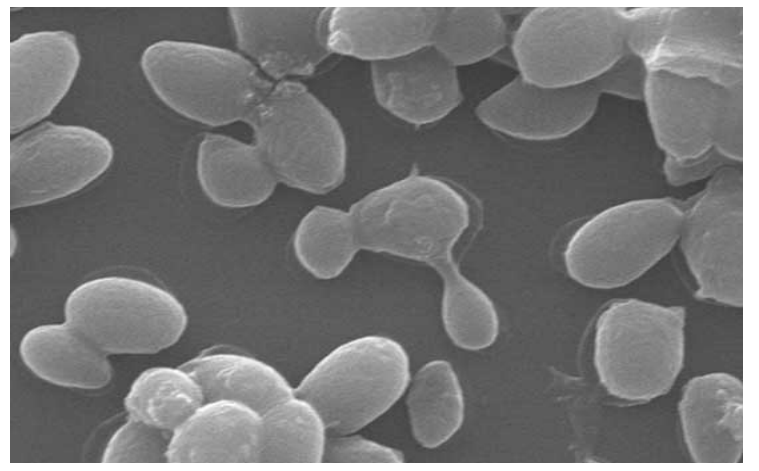

Fig. 2: Gemación de levaduras observada en un microscopio SEM con 5000 aumentos.

\section{CONCLUSIONES}

El experimento de fermentación propuesto brinda un nexo directo para que los estudiantes puedan entender intuitivamente procesos comunes encontrados en diversas industrias o en la vida diaria, como son los procesos de leudado de masa de harina o la producción de bebidas alcohólicas.

\section{REFERENCIAS}

Bravo B., M.A. Pesa y J.I. Pozo; The learning of science: a gradual change in the way of learning. The case of vision, Inv. Ensino Ciências: 14, 299-317 (2009).

Braun R. y V. Moses; A public policy on biotechnology education: what might be relevant and effective?, Current Opinion in Biotech.: 15, 246-249 (2004).

Harrigan M.T.; Industrial biotechnology education: A model of collaboration between industry and academia, Biochem. Mol. Biol. Educ.: 31, 142-144 (2003).

Jenkins R.O.; On the merits of viable-cell immobilization in biotechnology education, Biochem. Mol. Biol. Educ.: 28, 221-222 (2000).

Madigan M.T., J.M. Martinko, P.V. Dunlap y D.P. Clark; Brock Biology of Microorganisms: International Edition, Pearson Higher Education (2009).

Rota G. y J. Izquierdo; "Comics" as a tool for teaching biotechnology in primary schools, Electronic J. Biotech.: 6, No. 2 (2003).

Winters N. e Y. Mor; IDR: A participatory methodology for interdisciplinary design in technology enhanced learning, Computers \& Education: 50, 579-600 (2008). 